\title{
Biomimetic Dentistry using Stamp Technique for Direct Posterior Composite Restorations: A Case Report
}

\section{Sharma Shikha*}

Conservative Dentistry and Endodontics, Dental Department, E.S.I. Hospital, Amritsar, India

*Corresponding Author: Sharma Shikha, Conservative Dentistry and Endodontics, Dental Department, E.S.I. Hospital, Amritsar, India.

Received: July 19, 2019; Published: August 27, 2019

DOI: $10.31080 /$ ASDS.2019.03.0624

\begin{abstract}
"Stamp Technique" is a relatively novel and simple method for restoring carious teeth with intact occlusal topography, duplicating the occlusal anatomy with near perfection. This technique is highly precise and accurate when restoring functional occlusion. The purpose of this article is to demonstrate and discuss various aspects and applications of this technique, emphasising the fact that Stamp Technique enables the clinician to accomplish more in less time. A copy of the original unprepared tooth structure is used to replicate occlusal anatomy to get perfect anatomy in just a few minutes.
\end{abstract}

Keywords: Stamp Technique; Occlusion; Composite Resin

\section{Introduction}

Over the past few years, the demand for aesthetic restorations has increased considerably. As a result, newer techniques are being introduced and existing ones being refined continuously.

With the evolution of adhesive dentistry and propagation of concepts of minimal invasive dentistry, the predictability of composite restorations has been on the rise. However, achieving cusp-fossa relationship with adjacent teeth and creating occlusal harmony is a challenge in posterior composite restorations requiring both operator's skill as well as precious clinical time. Moreover, final polishing and adjustments is all the more tedious and taxing on operator's time especially in a busy practice scenario.

These issues are easily resolved with relatively novel and simple "Stamp Technique". This technique is useful in restoring carious teeth presenting with intact occlusal anatomy and marginal ridges with undermined caries [1]. Essentially, an index of the occlusal anatomy is created before initiating tooth preparation [2]. It is then pressed against the final uncured composite increment in the restoration after which composite is cured. This creates an exact replica of the pre-operative anatomy resulting in minimal requirement of finishing and final adjustments. Additionally, it provides the benefits of excluding air during final curing and creating minimal voids at occlusal surface.

As a result of exact reproduction of detailed occlusal anatomy, this technique falls under the realm of Biomimetic Dentistry which means 'mimicking nature' [3].

\section{Case 1}

A 23 year old female reported to the clinic complaining of mild sensitivity to cold and sweet in the lower back tooth region.

An oral examination revealed Class I caries on tooth 37 and 47(Figure 1). After thorough deliberation, it was decided to restore 47 and 37 using stamp technique.

A microbrush applicator tip was placed on tooth surface and flowable composite was placed, covering the tip and occlusal surface totally (Figure 2). Curing light was applied for 15 seconds. The cured index was removed from tooth surface and kept aside for later use. 


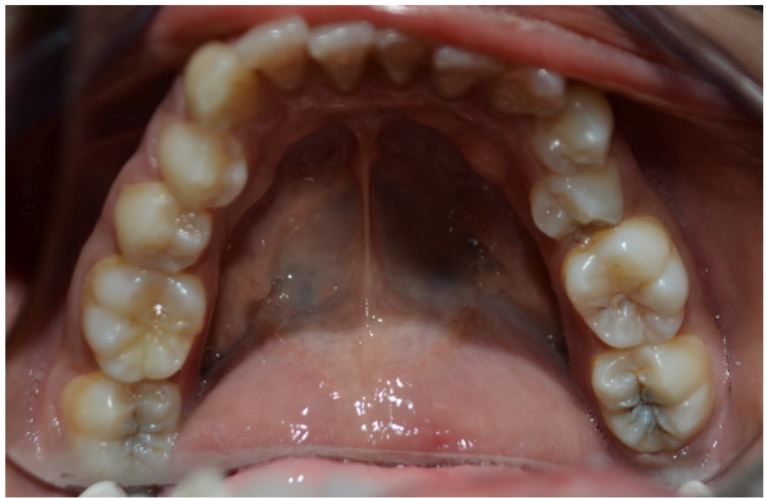

Figure 1: Pre Operative

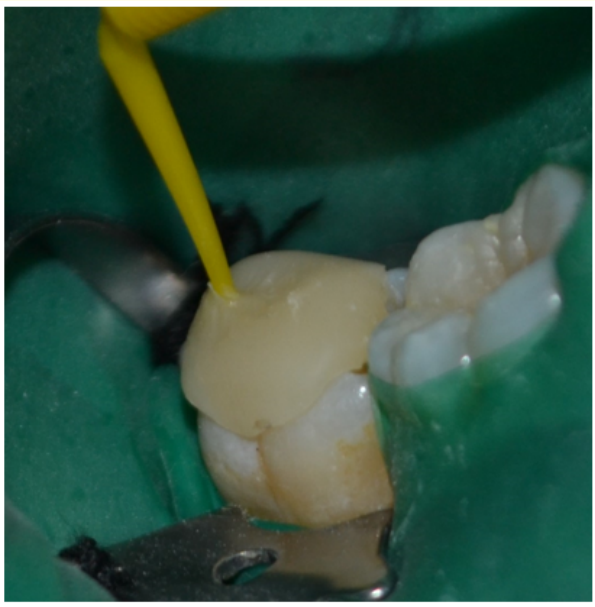

Figure 2: Fabrication of Stamp

Cavity preparation was initiated on the tooth (Figure 3). After caries removal the restorative process with composite resin was initiated under rubber dam isolation.

Acid etching was done using 37\% phosphoric acid (Figure 4). After rinsing and partially drying the tooth, bonding agent ( $\mathrm{G}$ bond, GC Asia Dental, Singapore) was applied according to manufacturer's instructions and cured.

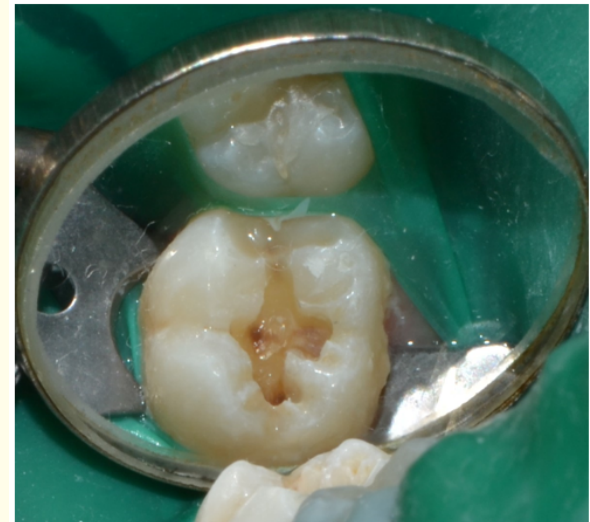

Figure 3: After Cavity Preparation

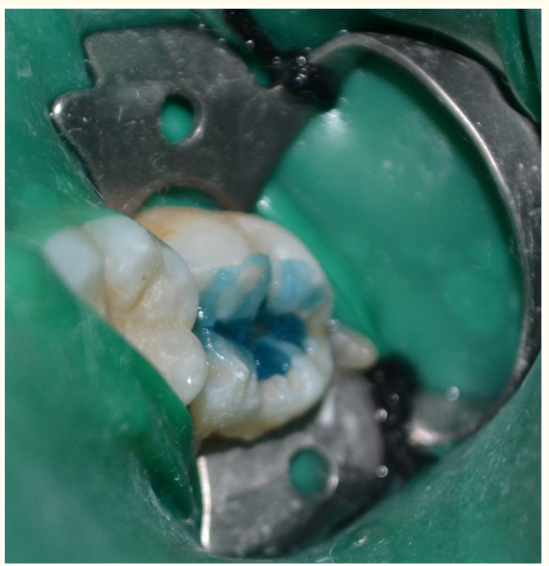

Figure 4: After Acid Etching

First increment of composite (Solare X, GC Asia Dental, Singapore) was placed, adapted to cavity floor and cured. After placing the second and final increment of composite, Teflon tape was placed as separator for index (Figure 5). Then index was placed and pressed on occlusal surface of tooth and curing was initiated. After 15 seconds curing, index and Teflon tape were removed and final curing was carried out (Figure 6). The excess composite was trimmed and finishing was carried out with soflex discs. 


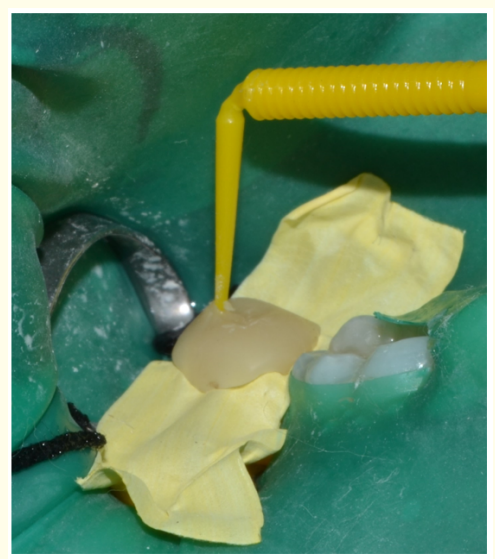

Figure 5: Placement of Final Increment with stamp in place separated by Teflon Tape

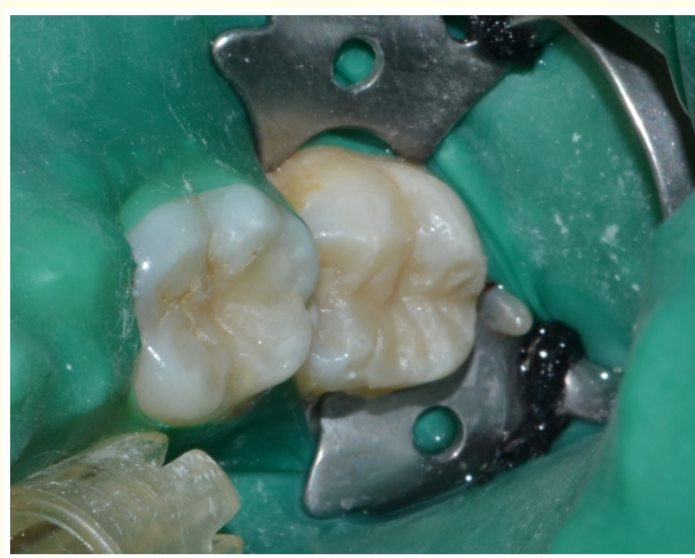

Figure 6: Immediate Post Operative

Figure 7 demonstrates the final restoration after checking with articulating paper. In this case a single shade of composite was used.

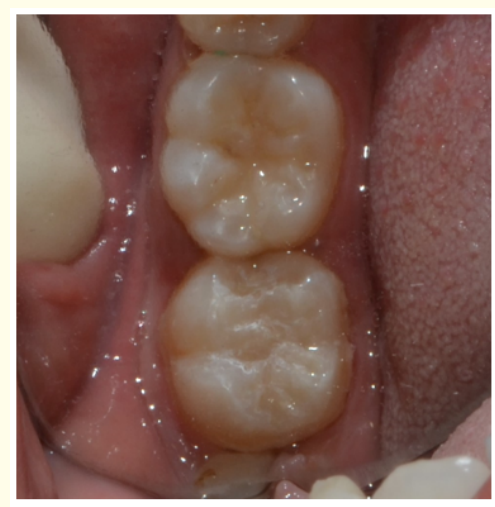

Figure 7: After final finishing

\section{Case 2}

The same patient had similar carious lesion on contralateral side. The same technique was applied to obtain an occlusal stamp and restore the tooth biomimetically.

Figures 8 to 15 demonstrate the various steps and completed restoration on 37 .

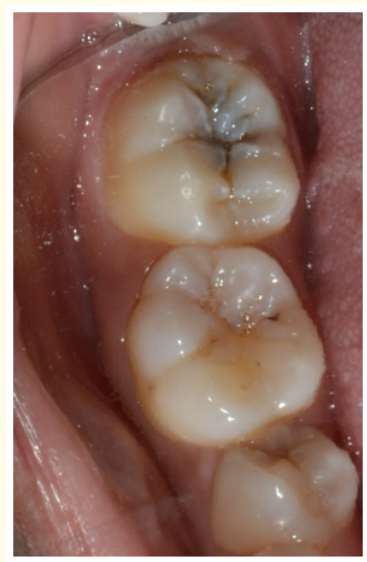

Figure 8: Pre Operative

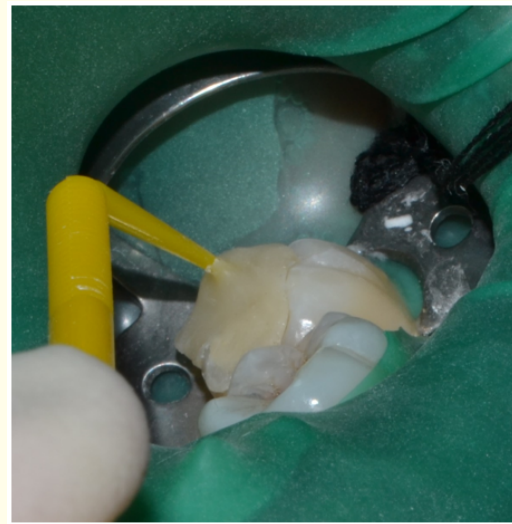

Figure 9: Fabrication of Occlusal Stamp

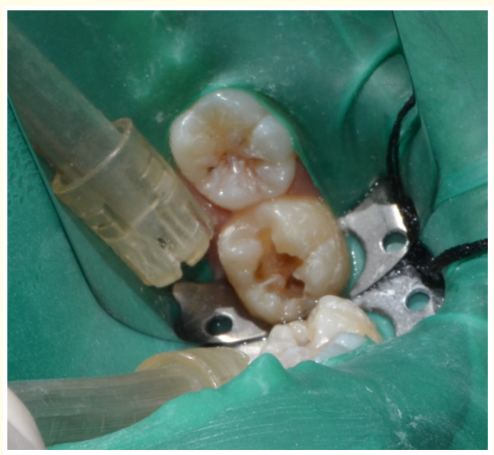

Figure 10: After Cavity Preparation 


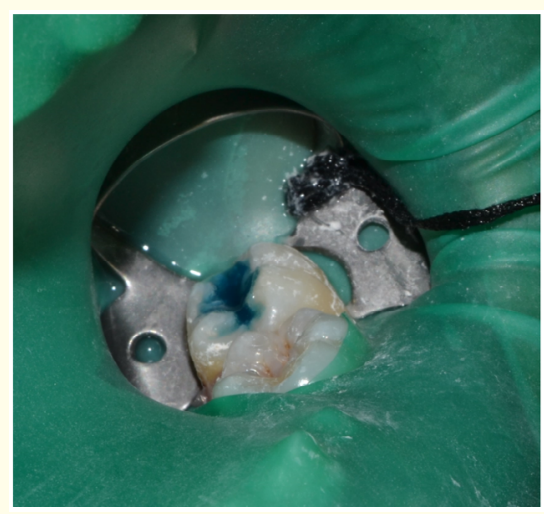

Figure 11: Acid Etching

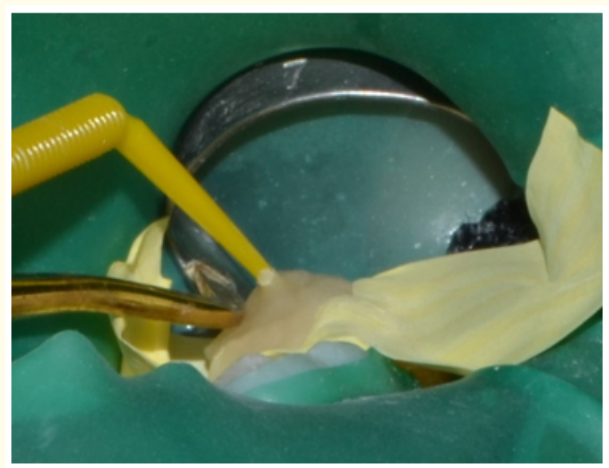

Figure 12: Placement of Final Increment

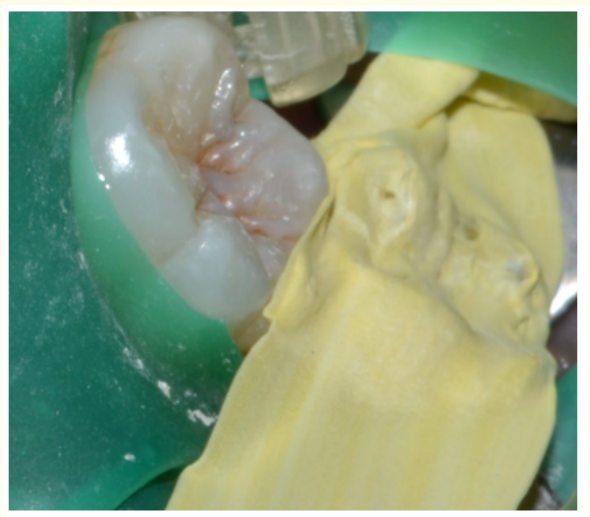

Figure 13: Occlusal Anatomy through Teflon Tape

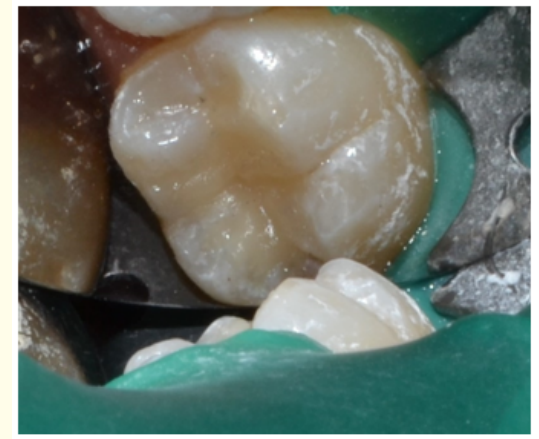

Figure 14: Immediate Post Operative

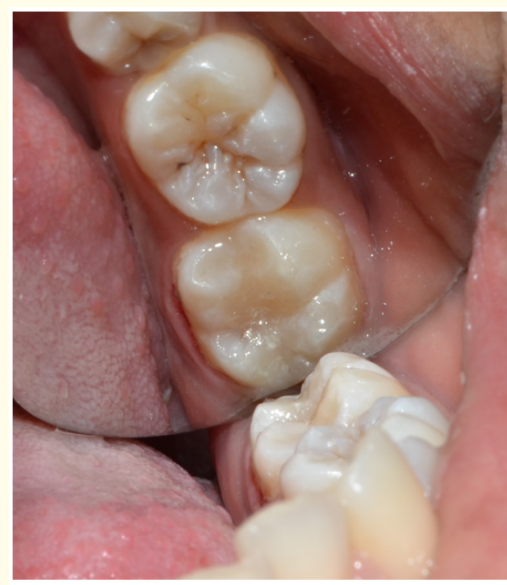

Figure 15: After Final Finishing

\section{Discussion}

Posterior teeth, at times, present with caries with intact enamel surface but undermined dentin. Sometimes, lesions referred to as “ Flouride Bombs" are present in a tooth [4]. The topical application of fluoridated agent seems to mask areas of dentin decay. Such lesions present as bluish/black discoloration under enamel surface or may be seen radiographically. These are cases suited for restorations using 'Stamp Technique'.

Certain other applications of 'Stamp Technique' include proximally carious endodontically involved teeth with absolutely intact occlusal topography. Stamps prepared before access opening, in such cases, may be utilized for restoring teeth with bonded 
restoration post obturation [3]. Also, it can be used indirectly in teeth having large carious lesions by preparing a stamp on the waxup model for the final bonded restoration [3].

Stamp can be prepared using flowable composites, or low viscosity composites. The expired composites may also be used for the fabrication of stamps [5].

In the given cases, no isolation agent has been used on tooth surface before fabrication of stamp. However, if the tooth presents with very deep pits and fissures using isolation agent is recommended. In such cases, the isolation agent fills the pits and fissures and does not allow subsequent flowable composite to flux inside. This leads to a more continuous surface of the final restoration.

Incremental technique of composite placement is used to avoid polymerisation shrinkage.

Teflon tape has been used with stamp technique which needs to be removed before final curing of composite resin. However, cling film can also be used instead of Teflon tape, in which case it does not need be removed before final curing.

Stamp technique has various advantages. Firstly, it helps in reducing overall time required for the restoration as instantly desired cusp -fossa relationship is attained [6]. This is suitable in a busy practice scenario as it ensures predictability with accuracy.

Secondly, degree of voids in final restoration is reduced. It helps in excluding oxygen from the top layer of composite and thus prevents its polymerisation inhibiting effect. This ensures long term success of the restoration [7].

The potential limitation of this technique is limited number of cases to which it can be applied. However, with modifications in the technique and further research, its scope can be expanded.

\section{Conclusion}

The accuracy offered is far greater than crafting of the restoration manually. It is a time saving, convenient, simple to master technique and a biomimetic procedure.

\section{Bibliography}

1. Pompeu JGF., et al. "Occlusal Stamp Technique for direct resin composite restoration: A Clinical Case Report”. International Journal of Recent Scientific Research 7 (2016): 12427-12430.

2. Alshehadat SA., et al. "The Stamp Technique for direct Class II composite restorations: A Case Series". Journal of Conservative Dentistry 19 (2016): 490-493.

3. Murashkin A. "Direct posterior composite restorations using stamp technique- Conventional and modified: A Case Report". International Journal of Dental Research 2 (2017): 3-7.

4. Page J. “The 'Fluoride syndrome': Occult caries?” British Dental Journal 160 (1986): 228-231.

5. Leibenberg WH. "Occlusal index-assisted restitution of aesthetic and functional anatomy in direct tooth-colored restorations". Quintessence International 27 (1996): 81-88.

6. Tambake NJ., et al. "Stamp Technique- New perspective of Aesthetic Dentistry: A Case Report". IOSRJ 16 (2017): 49-51.

7. Hamilton JC., et al. "Evaluation of custom occlusal matrix technique for posterior light-cured composites". Operative Dentistry 23 (1998): 303-307.

\section{Volume 3 Issue 9 September 2019}

(c) All rights are reserved by Sharma Shikha. 